\title{
MATÉRIA SECA E ACÚMULO DE NUTRIENTES EM GENÓTIPOS DE MILHO CONTRASTANTES QUANTO A AQUISIÇÃO DE FÓSFORO ${ }^{1}$
}

\author{
Dry matter and mineral nutrition in efficient corn genotypes \\ in relation to phosphurus acquisition
}

\author{
Edílson Carvalho Brasil², Vera Maria Carvalho Alves ${ }^{3}$, Ivanildo Evódio Marriel $^{3}$, \\ Gilson Villaça Exel Pitta ${ }^{3}$, Janice Guedes de Carvalho ${ }^{4}$
}

\begin{abstract}
RESUMO
Com o intuito de avaliar o efeito do estresse de P sobre atributos morfológicos do sistema radicular de genótipos de milho contrastantes quanto a eficiência na aquisição de fósforo, conduziu-se um experimento em casa-de-vegetação da Embrapa Milho e Sorgo, Sete Lagoas, MG, onde foram testados oito genótipos de milho, provenientes do programa de melhoramento da Empresa. O delineamento experimental utilizado foi o inteiramente casualizado, em esquema fatorial $8 \times 2$, com três repetições, correspondendo a oito genótipos (três linhagens: L1 = ineficiente, L2 e L3 = eficientes; e cinco híbridos: H1, H2 e H3 = eficientes, H4 e H5 = ineficientes) e dois níveis de $\mathrm{P}$ (baixo e alto). As plantas foram crescidas em solução nutritiva com duas concentrações de $\mathrm{P}$ equivalentes a $2,3 \mu \mathrm{M}$ e $129 \mu \mathrm{M}$. A composição da solução nutritiva foi a seguinte, em mg L ${ }^{-1}$ : $\mathrm{N}_{-} \mathrm{NO}_{3} 152 ; \mathrm{N}^{-\mathrm{NH}_{4}} 18,2 ; \mathrm{Ca} 141,1$; K 90,1; Mg 20,8; S 18,8; Fe 4,3; Mn 0,5; B 0,27; Cu 0,04; Zn 0,15; Mo 0,08; Na 0,04 e HEDTA 20,06. Aos 18 dias do transplantio, verificou-se que, sob condições de estresse de $\mathrm{P}$ em solução nutritiva, os híbridos $\mathrm{H} 5$, H1 e H2 apresentaram os maiores valores de massa seca total e da parte aérea, em relação aos demais. De modo geral, tanto os híbridos como as linhagens, quando foram crescidos em solução com baixo nível de $\mathrm{P}$, apresentaram maior relação raiz/parte aérea, do que os materiais que cresceram em solução com alto nível do nutriente. A concentração de $\mathrm{N}$ na parte aérea das plantas foi significativamente superior no tratamento com baixo nível de $\mathrm{P}$ na solução nutritiva. Os híbridos H1 e H2 e a linhagens L3 acumularam as maiores quantidades de P na parte aérea, independentemente do nível de P na solução. Esses resultados foram influenciados pelas maiores produções de produção de matéria seca apresentadas pelos referidos materiais. Independente do nível de P houve variação no conteúdo do nutriente na parte aérea dos híbridos, não sendo observado o mesmo comportamento para as linhagens.
\end{abstract}

Termos para indexação: Fósforo, eficiência, nutrição, genótipos.

\begin{abstract}
With the objective of evaluating the effect of phosphorus stress on morphological attributes of the root system of corn genotypes contrasting in relation to phosphorus efficiency, an experiment was carried out in nutrient solution in greenhouse at Embrapa Milho e Sorgo, Sete Lagoas, MG, in the months of november and december of 2001. The experiment design was totally at random, in an $8 \times 2$ factorial scheme with three repetitions, corresponding to eight genotypes (three lineages: L1 = inefficient, L2 and L3 = efficient, and five hybrids: $\mathrm{H} 1, \mathrm{H} 2$ and $\mathrm{H} 3=$ efficient, $\mathrm{H} 4$ and $\mathrm{H} 5=$ inefficient) and two phosphorus levels (low $=2.3 \mathrm{mM}$ and high $=129 \mathrm{mM}$ ). The seedlings were grown in nutrient solution with a following composition: in $\mathrm{mg} \mathrm{L}^{-1}: \mathrm{N}^{-\mathrm{NO}_{3}} 152 ; \mathrm{N}-\mathrm{NH}_{4} 18,2$; Ca 141,1; K 90,1; Mg 20,8; S 18,8; Fe 4,3; Mn 0,5; B 0,27; Cu 0,04; Zn 0,15; Mo 0,08; Na 0,04 e HEDTA 20,06. At 18 days after transplanting, the results showed that, under conditions of $\mathrm{P}$ stress in nutritive solution, the hybrids $\mathrm{H} 5, \mathrm{H} 1$ and $\mathrm{H} 2$ presented the largest values of total and shoot dry matter, in relation to the others. In general, the hybrids and inbred lines when were grown in solution with low level of $\mathrm{P}$, they presented larger root-shoot relationship, than the materials grew in solution with high level of the nutrient. The concentration of $\mathrm{N}$ in the shoot of the plants was superior in the treatment with low level of $\mathrm{P}$. The hybrids $\mathrm{H} 1$ and $\mathrm{H} 2$ and the inbred line L3 accumulated the largest amounts of $\mathrm{P}$ in the shoot, independently of the level of $\mathrm{P}$ in the solution. Independent of P level, there was variation in the nutrient content in the shoot of the hybrid, while in inbred lines were not observed the same behavior.
\end{abstract}

Index terms: Phosphorus, efficiency, nutrition, genotypes.

(Recebido em 21 de julho de 2004 e aprovado em 28 de dezembro de 2005)

\section{INTRODUÇÃo}

O milho tem grande importância econômica no Brasil, em razão das diversas formas de sua utilização, que vai desde a alimentação animal até a indústria de alta tecnologia, tendo expressiva participação na pauta de exportação nacional. Na safra 2003, a produção foi de aproximadamente 47 milhões de toneladas, o que coloca o País como o terceiro maior produtor mundial, ficando atrás somente dos Estados Unidos e China (FAO, 2004).

A maioria dos solos onde o milho tem sido cultivado são considerados pobres quimicamente, tendo como

Parte da tese de doutorado do primeiro autor - Universidade Federal de Lavras/UFLA - Cx. P. 3037 - 37.200-000 - Lavras, MG.

${ }^{2}$ Pesquisador Dr. da Embrapa Amazônia Oriental - Cx. P. 48 - 66.095-100 - Belém, PA - brasil@cpatu.embrapa.br

${ }^{3}$ Pesquisador Dr. da Embrapa Milho e Sorgo - Cx. P. 151 - 35.701-970 - Sete Lagoas, MG.

${ }^{4}$ Engenheiro Agrônomo., Dr. da Universidade Federal de Lavras/UFLA - Cx. P. 3037 - 37.200-000 - Lavras, MG 
características originais alta capacidade de sorção de $\mathrm{P}$, baixos teores de nutrientes, elevada acidez, resultando na baixa disponibilidade de P. Nessas áreas, para a obtenção de patamares de elevada produtividade da cultura, há necessidade do aumento do nível tecnológico empregado, ocasionando o aumento do uso de insumos, principalmente corretivos e fertilizantes, o que implica, no entanto, na elevação dos custos de produção.

Uma alternativa para minimizar esse problema seria o desenvolvimento de genótipos que apresentem boa produtividade, quando cultivados em solos com baixa fertilidade natural, especialmente naqueles que possuam baixos teores de P disponível. Nesse sentido, a obtenção de genótipos produtivos e com características de eficiência para absorver e utilizar $\mathrm{P}$, propicia maior eficiência no uso de fertilizantes fosfatados, melhorando a relação custo/ benefício ao longo do tempo, em decorrência do menor consumo desses insumos.

Diversos trabalhos têm mostrado que a eficiência de utilização de $\mathrm{P}$ pode ser aumentada em diversas culturas. No caso específico do milho, a diversidade inter e intraespecífica para a absorção, translocação, distribuição e uso de P, foi mostrada por Alves et al. (1988), Clark (1983), Schenk \& Barber (1979) e Silva \& Gabelman (1992).

O uso eficiente de nutrientes pelas plantas está relacionado à eficiência na aquisição, no transporte e na utilização de nutrientes que variam em função do genótipo e de fatores ambientais (BALIGAR \& FAGERIA, 1999; MARSCHNER, 1995). Diferenças genotípicas na eficiência a alguns nutrientes têm sido atribuídas a uma série de mecanismos, que estão relacionados às características de absorção, translocação e utilização de nutrientes pela planta, de forma isolada ou em conjunto (CLARK, 1983). Baker et al. (1970) observaram existir grandes diferenças, geneticamente controladas, na acumulação de $\mathrm{P}$ em híbridos de milho e que esse acúmulo não estava relacionado com a habilidade da planta em extrair $\mathrm{P}$ de solo com baixo nível do nutriente. Os autores concluíram que o teor de $\mathrm{P}$ em seedlings de milho poderia ser usado para diagnostico de disponibilidade de $\mathrm{P}$ no solo.

O objetivo deste trabalho foi avaliar a produção de matéria seca e o estado nutricional de genótipos de milho contrastantes quanto a eficiência para $\mathrm{P}$, cultivados em solução nutritiva.

\section{MATERIAL E MÉTODOS}

O experimento foi realizado nos meses de novembro e dezembro de 2001, em casa-de- vegetação da Embrapa Milho e Sorgo, em Sete Lagoas - MG, onde foram testados oito genótipos de milho, provenientes do programa de melhoramento da Empresa. Os materiais foram selecionados a partir de resultados de ensaios de campo, realizados anteriormente, onde as plantas foram caracterizadas quanto à eficiência para $\mathrm{P}$, com base na produção de grãos.

$\mathrm{O}$ delineamento experimental utilizado foi o inteiramente casualizado, em esquema fatorial $8 \times 2$, com três repetições. Os tratamentos corresponderam à combinação de oito genótipos e dois níveis de $\mathrm{P}$ (baixo e alto). Os genótipos utilizados foram: três linhagens (L1 ineficiente, L2 e L3 - eficientes) e cinco híbridos (H1 e H3 - híbridos simples eficientes, $\mathrm{H} 2$ - híbrido triplo eficiente, H4 e H5 - híbridos simples ineficientes). Os níveis de $\mathrm{P}$ corresponderam às concentrações de 2,3 $\mu \mathrm{M}$ e $129 \mathrm{M}$ de $\mathrm{P}$, que foram estabelecidos a partir de ensaios preliminares, realizados com base na produção de matéria seca esperada.

As sementes dos genótipos de milho foram desinfectadas com hipoclorito de sódio $(0,5 \%$ por 10 minutos) e germinadas em rolos de papel toalha colocados em vasos de plástico contendo água deionizada, constantemente aerada, ficando por quatro dias. Após a germinação, plântulas uniformes foram selecionadas, para posterior retirada do endosperma remanescente nas sementes, visando eliminar as reservas nutricionais da planta e garantir o estresse de $\mathrm{P}$ nos tratamentos com baixo nível do nutriente.

O experimento foi realizado conforme metodologia descrita por Schwarz \& Geisler (1991), em que as plantas foram cultivadas em solução nutritiva de Clark (1982a), modificada por Magnavaca (1982).

A composição da solução nutritiva foi a seguinte, em mg L-1: $\mathrm{N}^{-\mathrm{NO}_{3}}=152 ; \mathrm{N}^{-N_{4}}=18,2 ; \mathrm{Ca}=141,1 ; \mathrm{K}=90,1$; $\mathrm{Mg}=20,8 ; \mathrm{S}=18,8 ; \mathrm{Fe}=4,3 ; \mathrm{Mn}=0,5 ; \mathrm{B}=0,27 ; \mathrm{Cu}=0,04$; $\mathrm{Zn}=0,15 ; \mathrm{Mo}=0,08 ; \mathrm{Na}=0,04$ e HEDTA $=20,06$. Utilizaramse os seguintes sais: $\mathrm{Ca}\left(\mathrm{NO}_{3}\right)_{2} \cdot 4 \mathrm{H}_{2} \mathrm{O}, \mathrm{NH}_{4} \mathrm{NO}_{3}, \mathrm{KCl}, \mathrm{K}_{2} \mathrm{SO}_{4}$, $\mathrm{KNO}_{3}, \mathrm{Mg}\left(\mathrm{NO}_{3}\right)_{2} \cdot 6 \mathrm{H}_{2} \mathrm{O}, \mathrm{Fe}\left(\mathrm{NO}_{3}\right)_{3} \cdot 9 \mathrm{H}_{2} \mathrm{O}, \mathrm{MnCl}_{2} \cdot 4 \mathrm{H}_{2} \mathrm{O}$, $\mathrm{H}_{3} \mathrm{BO}_{3}, \mathrm{ZnSO}_{4} .7 \mathrm{H}_{2} \mathrm{O}, \mathrm{CuSO}_{4} \cdot 5 \mathrm{H}_{2} \mathrm{O}, \mathrm{Na}_{2} \mathrm{MoO}_{4} \cdot 2 \mathrm{H}_{2} \mathrm{O}$.

$\mathrm{O} \mathrm{P}$ foi adicionado na forma de $\mathrm{KH}_{2} \mathrm{PO}_{4}$, de acordo com as respectivas concentrações dos tratamentos. No tratamento correspondente ao baixo nível de $\mathrm{P}$, efetuou-se a complementação de $\mathrm{K}$, na forma de $\mathrm{KCl}$, para promover o balanceamento da solução nutritiva. $\mathrm{O}$ pH das soluções nutritivas foi ajustado para 5,5 e as soluções foram trocadas a cada quatro dias.

Para uniformizar as quantidades de nutrientes advindas das sementes, nos dois primeiros dias do experimento, todas as plantas foram mantidas em solução com alta concentração de $\mathrm{P}$, efetuando-se, em seguida, a aplicação dos tratamentos. 
Aos 18 dias do transplantio, as plantas foram colhidas e separadas em raiz e parte aérea e colocadas em estufa de circulação forçada de ar, a aproximadamente $70^{\circ}$ $\mathrm{C}$, até obtenção de peso constante. Após secagem, o material vegetal foi pesado para obtenção da produção de matéria seca e moído para determinação dos teores de N, P, $\mathrm{K}, \mathrm{Ca}, \mathrm{Mg}$ no tecido vegetal, conforme metodologia descrita por Sarruge \& Haag (1974).

Os resultados de produção de matéria seca e de nutrição mineral referentes aos híbridos e linhagens foram submetidos à análise de variância (teste F) separadamente, em decorrência das características de vigor desses materiais e, conforme a significância dos resultados, efetuou-se a comparação das médias dos tratamentos pelo teste de ScottKnott, ao nível de 5\% de probabilidade.

\section{RESULTADOS E DISCUSSÃO}

\section{Produção de matéria seca}

A produção de matéria seca da raiz dos híbridos de milho não diferiu significativamente entre os tratamentos. No entanto, a produção de matéria da parte aérea dos híbridos H5, H1 e H2 foram superiores aos demais, sob condições de estresse de $\mathrm{P}$ em solução nutritiva (Tabela 1). Entre as linhagens, a L3 (eficiente) destacou-se das demais por apresentar as maiores produções de matéria seca da raiz e da parte aérea (Tabela 2).

A diferença de produção de matéria seca entre os níveis baixo e alto pode ser um indicativo da capacidade dos híbridos de se desenvolverem normalmente em condições de estresse de P. Considerando-se esse aspecto, observou-se valores positivos para essa diferença na produção de matéria seca de raiz dos híbridos H1, H4 e H5 (Figura 1), indicando o maior crescimento de raiz com baixo P. Isso demonstra que os híbridos promoveram uma alteração fisiológica no aumento de matéria seca, em função da concentração de $\mathrm{P}$ no meio. Verificou-se que entre as linhagens, apenas a L3 apresentou comportamento semelhante.

Clark (1982b) menciona que plantas de milho sob deficiência de $\mathrm{P}$ apresentam menor crescimento da parte aérea, do que das raízes e estas parecem ter prioridade para utilizar o nutriente quando ele é inicialmente absorvido. Liu et al. (1998) assinalam que, quando a planta encontra-se sob estresse de $\mathrm{P}$, a distribuição, a direção e a energia de assimilação dos produtos da fotossíntese são direcionados para a formação e o desenvolvimento do sistema radicular, visando aumentar a área de absorção do nutriente. Segundo Clarkson (1985), as raízes tornam-se os drenos preferenciais de fotoassimilados, quando alguns nutrientes encontramse limitando o crescimento das plantas, especialmente $\mathrm{P}$ e N.

TABELA 1 - Produção de matéria seca da raiz, da parte aérea e total de híbridos de milho em função de níveis baixo (2,3 $\mu \mathrm{M})$ e alto $(129 \mu \mathrm{M})$ de $\mathrm{P}$ na solução nutritiva.

\begin{tabular}{|c|c|c|c|c|}
\hline \multirow[b]{2}{*}{ Genótipo } & \multicolumn{4}{|c|}{ Nível de $\mathbf{P}^{(1)}$} \\
\hline & \multicolumn{2}{|c|}{ Baixo } & Alto & \multirow[t]{2}{*}{ Média } \\
\hline & \multicolumn{3}{|c|}{ Matéria seca da parte aérea $\left(\mathrm{g}\right.$ planta $\left.{ }^{-1}\right)$} & \\
\hline $\mathrm{H} 1(\mathrm{E})$ & $0,229 \mathrm{a}$ & $\mathrm{B}$ & $0,271 \mathrm{c} \quad \mathrm{A}$ & $0,250 \mathrm{~ns}$ \\
\hline $\mathrm{H} 2(\mathrm{E})$ & 0,217 a & $\mathrm{B}$ & 0,375 a $\mathrm{A}$ & $0,296 \mathrm{~ns}$ \\
\hline H3 (E) & $0,170 \mathrm{~b}$ & B & $0,313 \mathrm{~b} \quad \mathrm{~A}$ & $0,241 \mathrm{~ns}$ \\
\hline $\mathrm{H} 4$ (I) & $0,166 \mathrm{~b}$ & $\mathrm{~B}$ & $0,332 \mathrm{~b} \quad \mathrm{~A}$ & $0,249 \mathrm{~ns}$ \\
\hline H5 (I) & 0,247 a & $\mathrm{B}$ & $0,322 \mathrm{~b} \quad \mathrm{~A}$ & $0,284 \mathrm{~ns}$ \\
\hline \multirow[t]{2}{*}{ Média } & $0,205 \mathrm{NS}$ & & $0,322 \mathrm{NS}$ & -- \\
\hline & \multicolumn{4}{|c|}{ Matéria seca total (g planta $\left.{ }^{-1}\right)$} \\
\hline $\mathrm{H} 1(\mathrm{E})$ & 0,413 a & A & 0,429 b A & $0,421 \mathrm{~ns}$ \\
\hline $\mathrm{H} 2(\mathrm{E})$ & 0,378 a & B & 0,559 a $\mathrm{A}$ & $0,468 \mathrm{~ns}$ \\
\hline H3 (E) & $0,331 \mathrm{~b}$ & B & $0,491 \mathrm{~b} \quad \mathrm{~A}$ & $0,411 \mathrm{~ns}$ \\
\hline H4 (I) & $0,312 \mathrm{~b}$ & B & $0,467 \mathrm{~b} \quad \mathrm{~A}$ & $0,389 \mathrm{~ns}$ \\
\hline H5 (I) & 0,426 a & A & $0,463 \mathrm{~b} \quad \mathrm{~A}$ & $0,444 \mathrm{~ns}$ \\
\hline Média & $0,372 \mathrm{NS}$ & & $0,482 \mathrm{NS}$ & -- \\
\hline
\end{tabular}

${ }^{(1)}$ Médias seguidas da mesma letra minúscula na vertical e maiúscula na horizontal não diferem significativamente pelo teste de Scott-Knott, ao nível de 5\%. I - ineficiente, E - eficiente, ns - não- significativo.

Ciênc. agrotec., Lavras, v. 31, n. 3, p. 704-712, maio/jun., 2007 
TABELA 2 - Produção de matéria seca da raiz, da parte aérea e total de linhagens de milho em função de níveis baixo $(2,3 \mu \mathrm{M})$ e alto $(129 \mu \mathrm{M})$ de $\mathrm{P}$ na solução nutritiva ${ }^{(1)}$.

\begin{tabular}{lccc}
\hline & \multicolumn{3}{c}{ Nível de P } \\
\cline { 2 - 4 } Genótipo & Baixo & Alto & Média \\
\hline & & Matéria seca da raiz $\left(\mathrm{g} \mathrm{planta}^{-1}\right)$ & \\
L1 (I) & $0,065 \mathrm{~ns}$ & $0,077 \mathrm{~ns}$ & $0,071 \mathrm{~b}$ \\
L2 (E) & $0,110 \mathrm{~ns}$ & $0,134 \mathrm{~ns}$ & $0,122 \mathrm{a}$ \\
L3 (E) & $0,135 \mathrm{~ns}$ & $0,120 \mathrm{~ns}$ & $0,128 \mathrm{a}$ \\
Média & $0,103 \mathrm{~ns}$ & $0,111 \mathrm{~ns}$ & -- \\
& & Matéria seca da parte aérea $\left(\mathrm{g} \mathrm{planta}^{-1}\right)$ & \\
L1 (I) & $0,138 \mathrm{~ns}$ & $0,168 \mathrm{~ns}$ & $0,153 \mathrm{~b}$ \\
L2 (E) & $0,130 \mathrm{~ns}$ & $0,191 \mathrm{~ns}$ & $0,160 \mathrm{~b}$ \\
L3 (E) & $0,192 \mathrm{~ns}$ & $0,241 \mathrm{~ns}$ & $0,216 \mathrm{a}$ \\
Média & $0,153 \mathrm{~B}$ & $0,200 \mathrm{~A}$ & -- \\
\hline
\end{tabular}

${ }^{(1)}$ Médias seguidas da mesma letra minúscula na vertical e maiúscula na horizontal não diferem significativamente pelo teste de Scott-Knott, ao nível de 5\%. I - ineficiente, E - eficiente, ns - não- significativo.

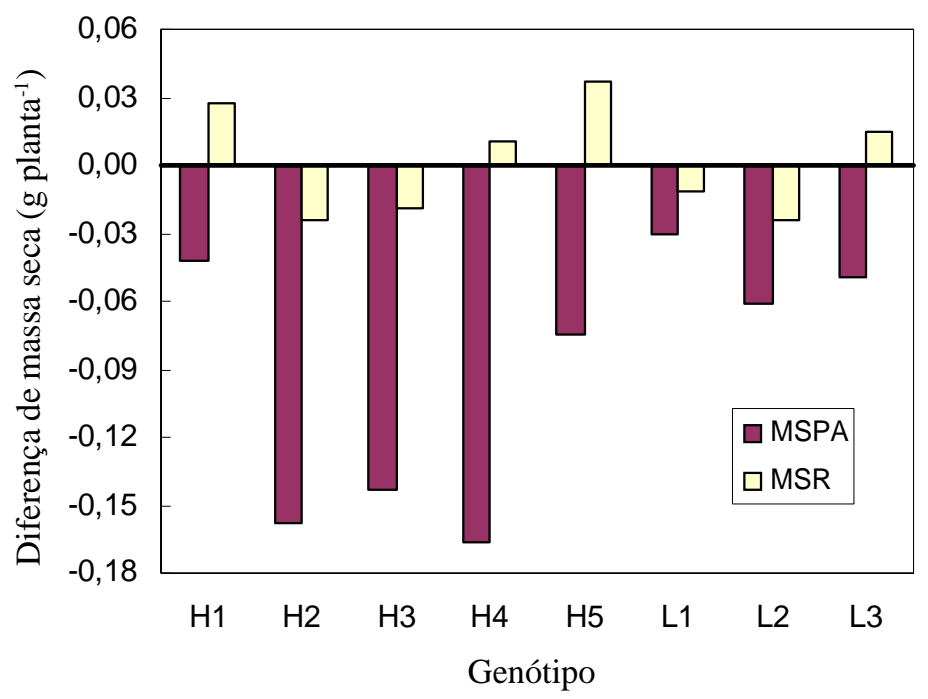

FIGURA 1 - Diferença de produção de matéria seca da raiz (MSR) e da parte aérea (MSPA) de genótipos de milho entre os níveis baixo e alto de fósforo em solução nutritiva.

Tanto os híbridos como as linhagens, crescidos em solução com baixo nível de P, apresentaram maior relação raiz/parte aérea, do que os materiais crescidos em solução com alto nível do nutriente, com exceção da linhagem L1, indicando que essa característica não apresenta uma resposta genética única para o milho (Tabela 3). De acordo com os resultados, as raízes das plantas de milho cultivadas em condições de insuficiência de $\mathrm{P}$, tenderam a apresentar um aumento proporcional de matéria seca, em relação à parte aérea, o que pode favorecer maior absorção do nutriente.

No baixo nível de $\mathrm{P}$, os híbridos $\mathrm{H} 3$ e $\mathrm{H} 4$ foram estatisticamente superiores aos demais, em termos de relação raiz/parte aérea, enquanto que entre as linhagens, a L2 foi superior às demais (Tabela 3 ). 
TABELA 3 - Relação de massa seca entre a raiz e a parte aérea de genótipos de milho em função dos níveis baixo $(2,3 \mu \mathrm{M})$ e alto $(129 \mu \mathrm{M})$ de $\mathrm{P}$ em solução nutritiva.

\begin{tabular}{cccc}
\hline \multirow{2}{*}{ Genótipo } & \multicolumn{4}{c}{ Relação raiz / parte aérea } \\
\cline { 2 - 4 } & P - baixo & P - alto & Média \\
\hline H1 (E) & 0,811 b A & 0,578 a B & $0,694 \mathrm{~ns}$ \\
H2 (E) & 0,745 b A & 0,495 a B & $0,620 \mathrm{~ns}$ \\
H3 (E) & 0,947 a A & 0,571 a B & $0,759 \mathrm{~ns}$ \\
H4 (I) & 0,873 a A & 0,407 b B & $0,640 \mathrm{~ns}$ \\
H5 (I) & 0,722 b A & 0,440 b B & $0,581 \mathrm{~ns}$ \\
Média & 0,819 NS & 0,498 NS & -- \\
& & Linhagem & $0,463 \mathrm{~ns}$ \\
L1 (I) & 0,463 b A & 0,464 b A & $0,772 \mathrm{~ns}$ \\
L2 (E) & 0,839 a A & 0,705 a B & $0,604 \mathrm{~ns}$ \\
L3 (E) & 0,708 a A & 0,501 b B & - \\
Média & 0,670 NS & 0,557 NS & \\
\hline
\end{tabular}

${ }^{(1)}$ Médias seguidas da mesma letra minúscula na vertical e maiúscula na horizontal não diferem significativamente pelo teste de Scott-Knott, ao nível de 5\%. I - ineficiente, E - eficiente, ns - não- significativo.

Considerando-se a diferença dos valores da relação raiz/parte aérea entre os tratamentos com baixo e alto de $\mathrm{P}$, observou-se que o híbrido H4 apresentou maior variação, em relação aos demais, representando uma diferença da ordem de $114 \%$ superior, na condição de estresse de P. A menor variação foi observada para o híbrido $\mathrm{H} 1$, com aumento de $40 \%$ na relação raiz/parte aérea. Entre as linhagens, a L3 foi a que apresentou maior variação, com aumento de $41 \%$ no tratamento com baixo nível de $\mathrm{P}$, em relação ao alto nível de $\mathrm{P}$.

Vários autores têm observado essa tendência de aumento da relação de massa seca entre a raiz e a parte aérea, para diversas espécies, sob condições de deficiência de P (ALVES et al., 1988; HALSTED \& LYNCH, 1996; MACHADO, 1995). Segundo Gill et al. (1992), esse processo pode ser explicado pela mobilização de carboidratos na planta. Conforme os autores, em condições sub-ótimas de $\mathrm{P}$ para as plantas, geralmente, o conteúdo foliar de carboidratos é aumentado, já que a quantidade de fotossintatos produzida nessas condições é maior do que poderia ser utilizada para o crescimento da parte aérea. Então, os carboidratos em excesso são translocados e acumulados nas raízes, induzindo uma utilização desproporcional do substrato, resultando em maior taxa de crescimento das raízes em relação à parte aérea.

\section{Acúmulo de nutrientes}

Quando as plantas cresceram em solução com baixo nível de $\mathrm{P}$, observaram-se menores valores no conteúdo de N na parte aérea dos genótipos (Tabela 4). Esse aspecto também foi demonstrado por outros autores (ALVES et al., 1996, 1999; ANDREEVA et al., 1992; MAGALHÃES, 1995), que verificaram uma redução no conteúdo de $\mathrm{N}$ na parte aérea das plantas, com a omissão de $\mathrm{P}$ na solução nutritiva. Os híbridos não diferiram nas quantidades de $\mathrm{N}$ na parte aérea. Dentre as linhagens, a L3 apresentou maior acúmulo de $\mathrm{N}$ na parte aérea, em relação às demais. Houve pouca variação entre os conteúdos de $\mathrm{N}$ nas raízes dos híbridos, observando-se que somente o $\mathrm{H} 4$ foi inferior aos demais (Tabela 5). As linhagens L2 e L3 apresentaram maiores quantidades de $\mathrm{N}$ acumuladas nas raízes, em relação à $\mathrm{L} 1$.

Os híbridos $\mathrm{H} 1$ e $\mathrm{H} 2$ acumularam as maiores quantidades de $\mathrm{P}$ na parte aérea, independentemente do nível de P na solução (Tabela 4). Dentre as linhagem, a L3 foi superior às demais. Esses resultados foram influenciados pelas maiores produções de matéria seca apresentadas pelos referidos materiais. $\mathrm{O}$ conteúdo de $\mathrm{P}$ nas raízes não diferiu entre os genótipos, independente do nível de P na solução (Tabela 5). No entanto, observou-se que os genótipos crescidos com alto nível de $\mathrm{P}$ em solução apresentaram maiores valores de $\mathrm{P}$ absorvido, do que aqueles com baixo P. Resultados semelhantes foram obtidos por Machado et al. (1999), testando a variabilidade de genótipos de milho para eficiência de uso de P. 


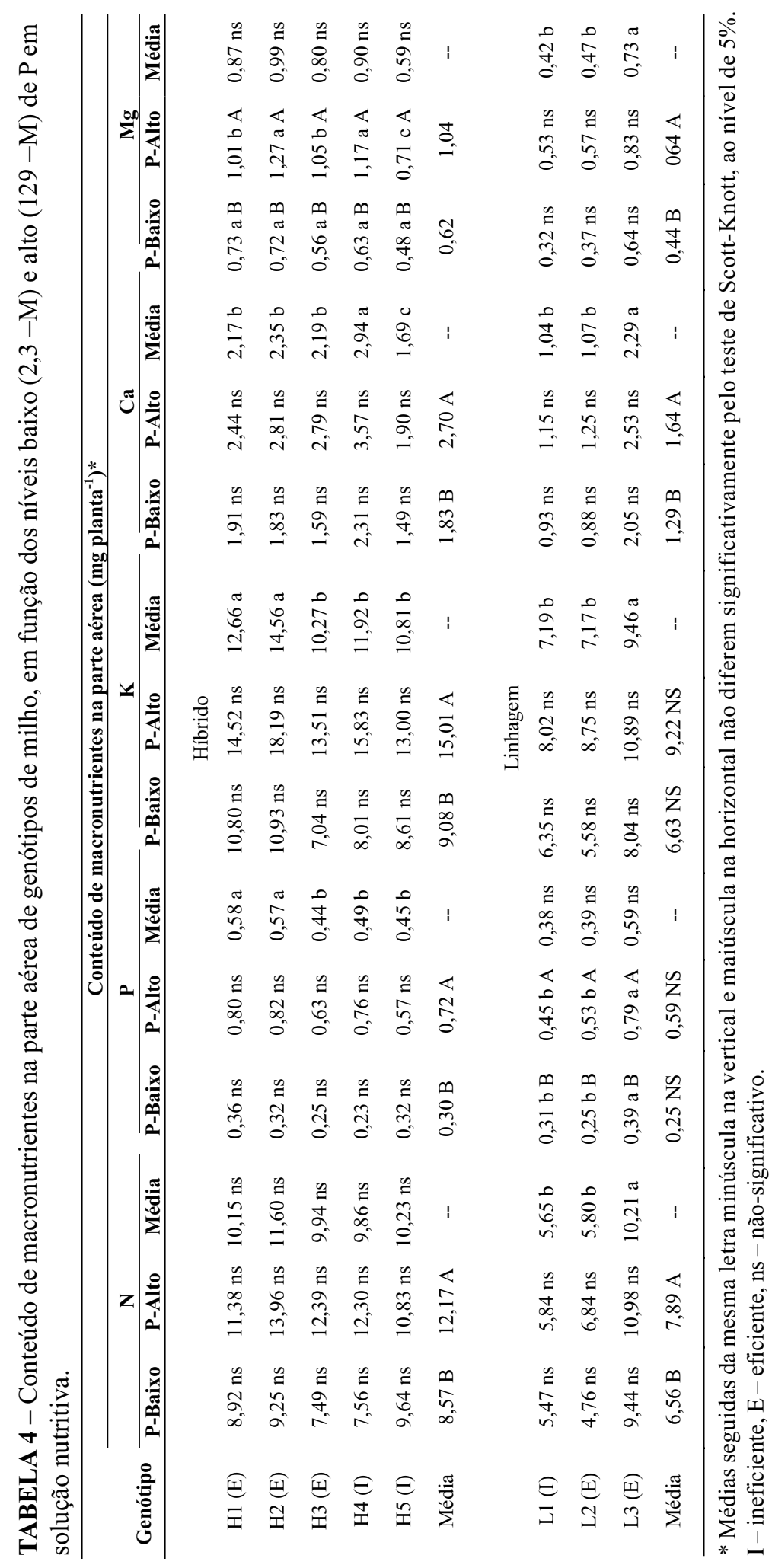

Ciênc. agrotec., Lavras, v. 31, n. 3, p. 704-712, maio/jun., 2007 


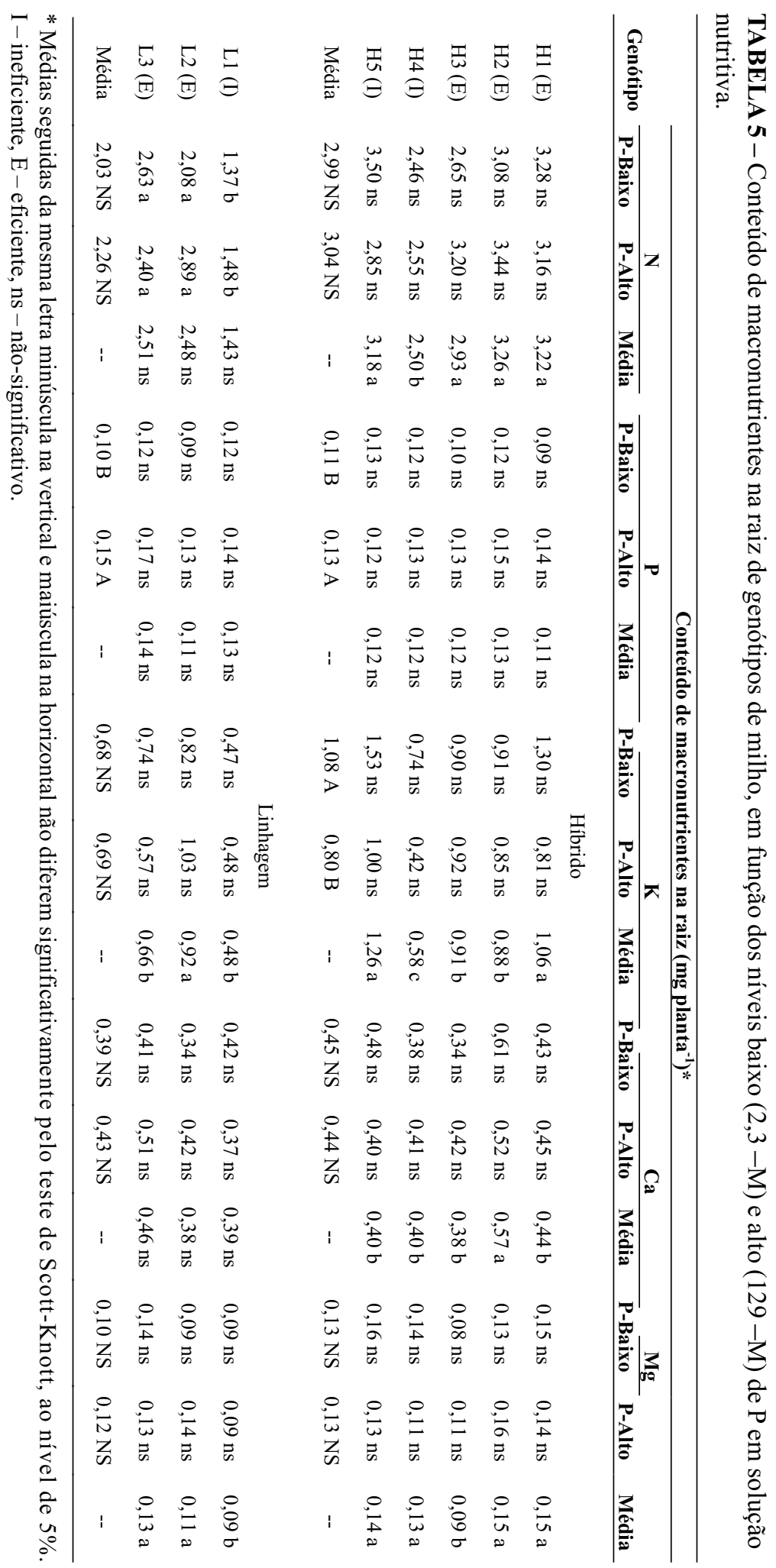

Ciênc. agrotec., Lavras, v. 31, n. 3, p. 704-712, maio/jun., 2007 
As maiores quantidades de $\mathrm{K}$ na parte aérea foram encontradas nos híbridos $\mathrm{H} 1$ e $\mathrm{H} 2$, que apresentaram valores significativamente superiores aos demais, independentemente do nível de $\mathrm{P}$ no meio (Tabela 4). O nível de $\mathrm{P}$ na solução favoreceu o aumento do conteúdo de $K$ na parte aérea de todos os genótipos. Comportamento oposto foi verificado nas raízes, em que o teor do nutriente foi, em média, menor, no maior nível de P na solução (Tabela 5).

$\mathrm{O}$ alto nível de $\mathrm{P}$ em solução favoreceu o acúmulo de $\mathrm{Ca}$ e $\mathrm{Mg}$ na parte aérea dos genótipos, efeito esse que não foi observado nas raízes, onde não houve efeito de níveis de $\mathrm{P}$ (Tabelas 4 e 5). O conteúdo de $\mathrm{Mg}$ na parte aérea dos híbridos não variou, quando foram crescidos sob estresse de P (Tabela 4).

Com relação ao $\mathrm{P}$ total na planta, os genótipos crescidos em alto nível de $\mathrm{P}$ apresentaram quantidades significativamente maiores, do que os materiais que cresceram em solução com baixo nível do nutriente (Tabela 6). Apesar das diferenças observadas no conteúdo de $\mathrm{P}$ na parte aérea, as quantidades de $\mathrm{P}$ total não diferiram entre os híbridos cultivados em solução de crescimento com baixo nível de $\mathrm{P}$.

TABELA 6 - Quantidades de P total acumuladas por genótipos de milho, em função de níveis baixo $(2,3 \mu \mathrm{M})$ e alto $(129 \mu \mathrm{M})$ de $\mathrm{P}$ em solução nutritiva.

\begin{tabular}{|c|c|c|}
\hline \multirow{2}{*}{ Genótipo } & \multicolumn{2}{|c|}{ P total acumulado (mg planta $\left.{ }^{-1}\right)$} \\
\hline & Baixo P & Alto P \\
\hline & \multicolumn{2}{|c|}{ Híbrido } \\
\hline H1 (E) & 0,46 a $\mathrm{B}$ & 0,95 a $\mathrm{A}$ \\
\hline $\mathrm{H} 2(\mathrm{E})$ & 0,45 a $\mathrm{B}$ & 0,97 a $\mathrm{A}$ \\
\hline H3 (E) & 0,35 a $\mathrm{B}$ & $0,77 \mathrm{~b} A$ \\
\hline H4 (I) & 0,35 a $\mathrm{B}$ & 0,90 a $A$ \\
\hline \multirow[t]{2}{*}{ H5 (I) } & 0,46 a $\mathrm{B}$ & $0,70 \mathrm{~b} A$ \\
\hline & \multicolumn{2}{|c|}{ Linhagem } \\
\hline L1 (I) & 0,41 b B & 0,59 b A \\
\hline L2 (E) & $0,34 \mathrm{~b}$ B & $0,66 \mathrm{~b} \quad \mathrm{~A}$ \\
\hline L3 (E) & 0,51 a $\mathrm{B}$ & 0,97 a $\mathrm{A}$ \\
\hline
\end{tabular}

${ }^{(1)}$ Médias seguidas da mesma letra minúscula na vertical e maiúscula na horizontal não diferem significativamente pelo teste de Scott-Knott, ao nível de 5\%. I - ineficiente, $\mathrm{E}$-eficiente.
Em ambos os níveis de P em solução, a linhagem L3 apresentou quantidade superior de P total acumulado, em relação às demais. Pode-se observar que as quantidades de $\mathrm{P}$ total acumuladas por essa linhagem foram superiores às dos híbridos, em condições de baixo nível de $\mathrm{P}$, e igualaram-se às dos híbridos crescidos sob alto nível do nutriente na solução. Os resultados indicam a alta capacidade dessa linhagem na absorção do nutriente, independentemente do nível de $\mathrm{P}$ no meio.

\section{CONCLUSÕES}

De acordo com os resultados obtidos pode-se concluir que:

- Sob condições de estresse de $\mathrm{P}$, as produções de matéria seca da parte aérea e total dos híbridos de milho foram variáveis, não havendo o mesmo comportamento para as linhagens;

- A insuficiência do P no meio favoreceu o aumento da relação raiz / parte aérea em todos os genótipos;

- Independente do nível de P houve variação no conteúdo do nutriente na parte aérea dos híbridos, não sendo observado o mesmo comportamento para as linhagens.

\section{REFERÊNCIAS BIBLIOGRÁFICAS}

ALVES, V. M. C.; MAGALHÃES, J. V.; VASCONCELOS, R. F.; NOVAIS, R. F.; BAHIA FILHO, A. F. C.; FRANÇA, G. E.; OLIVEIRA, C. A.; FRANÇA, C. C. M. Acúmulo de nitrogênio e de fósforo em plantas de milho afetadas pelo suprimento parcial de fósforo de raízes. Revista Brasileira de Ciência do Solo, Viçosa, v. 23, n. 2, p. 299-305, abr./jun. 1999.

ALVES, V. M. C.; NOVAIS, R. F.; OLIVEIRA, M. F. G.; BARROS, N. F. Efeito da omissão de fósforo na absorção de nitrogênio por híbridos de milho (Zea mays, L.). Revista Ceres, Viçosa, v. 43, n. 248, p. 435-443, jul./ago. 1996.

ALVES, V. M. C.; NOVAIS, R. F. de; OLIVEIRA, M. F. G. de; SANT'ANNA, R. Cinética e translocação de fósforo em híbridos de milho. Pesquisa Agropecuária Brasileira, Brasília, v. 33, n. 7, p. 1047-1052, jul. 1988.

ANDREEVA, T. F.; MAEVSKAYA, S. N.; VOEVUDSKAYA, S. Y. Interrelationship between photosynthesis and nitrogen metabolism in mus tarda plantsonder condictions of different phosphorus and nitrogen nutrition. Soviet Plant Physiology, Moscow, v. 39, n. 2, p. 438-442, Mar./ Apr. 1992. 
BAKER, D. E.; JARREL, A. E.; MARSHAL, L. E.; THOMAS, W. I. Phosphorus uptake from soils by corn hybrids selected for high and low phosphorus accumulation. Agronomy Journal, Madison, v. 62, p. 103-106, 1970.

BALIGAR, V. C.; FAGERIA, N. K. Plant nutrient efficiency: forward the second paradigm. In: SIQUEIRA, J.; MOREIRA, F. M. S.; LOPES, A. S.; GUILHERME, L. R. G.; FAQUIN, V.; FURTINI NETO, A. E.; CARVALHO, J. G. de. Inter-relação fertilidade, biologia do solo e nutrição de plantas. Lavras: UFLA/DCS/SBCS, 1999. p. 183-204.

CLARK, R. B. Nutrient solution growth of sorghum and corn in mineral nutrition studies. Journal of Plant Nutrition, New York, v. 5, n. 8, p. 1039-1057, 1982a.

CLARK, R. B. Plant response to mineral element toxicity and deficiency. In: CHRISTIANSEN, M. N.; LEWIS, C. F. (Eds.). Breeding plants for less favorable environments. New York: J. Wiley \& Sons, 1982b. p. 71-73.

CLARK, R. B. Plant genotype differences in the uptake, translocation, accumulation and use of mineral elements required for plant growth. In: SARIC, M. R.; LONGHMANN, B. C. (Eds.). Genetic aspects of plant nutrition. The Hague: Martinus Nighoff, 1983. p. 49-70.

CLARKSON, D. T. Adaptações morfológicas e fisiológicas das plantas a ambientes de baixa fertilidade. In: REUNIÃO DE FERTILIDADE DO SOLO, 16., 1985, Ilhéus. Anais... Ilhéus: P. Cabala Rosand, 1985. p. 45-75.

FAO. Database gateway FAOSTAT. Disponível em: <http:\|www.fao.org>. Acesso em: 12 mar. 2004.

GILL, M. A.; SALIM, R. M.; ZIA, M. S. Maize growth and uptake of phosphorus and copper at different ambient phosphate concentrations. Soil Science and Plant Nutrition, Tokyo, v. 38, n. 4, p. 631-636, Dec. 1992.

HALSTED, M.; LYNCH, J. Phosphorus responses of C3 and C1 species. Journal of Experimental Botany, Oxford, v. 47, n. 297, p. 497-505, Apr. 1996.
LIU, G.; LI, J.; LI, Z. Effect from horizontally diving the root system of weat plants having different phosphorus efficiencies. Journal of Plant Nutrition, New York, v. 21, n. 12, p. 2535-2544, 1998.

MACHADO, C. T. de T. Avaliação da eficiência de utilização de fósforo de variedades locais de milho (Zea mays l.). 1995. 131 f. Dissertação (Mestrado) - Universidade Federal Rural do Rio de Janeiro, Itaguaí, 1995.

MACHADO, C. T. de T.; GUERRA, J. G. M.; ALMEIDA, D. L. de; MACHADO, A. T. Variedade entre genótipos de milho para eficiência em níveis de fósforo. Bragantia, Campinas, v. 58, n. 1, p. 109-124, 1999.

MAGALHÃES, J. V.Alterações na absorção e no metabolismo do nitrigênio em diferentes níveis de estresse de fósforo em plantas de milho (Zea mays, L.). 1995. 76 f. Dissertação (Mestrado) - Universidade Federal de Viçosa, Viçosa, 1995.

MAGNAVACA, R. Genetic variability and the inheritance of aluminum tolerance in mayze (Zea mays L.). 1982. Thesis (Ph.D) - University Lincoln, Nebrasca, 1982.

MARSCHNER, H. Mineral nutrition of hight plants. San Diego: Academic, 1995. 889 p.

SARRUGE, J. R.; HAAG, H. P. Análises químicas em plantas. Piracicaba: ESALQ/USP, 1974. 57 p.

SCHENK, M. K.; BARBER, S. A. Phosphate uptake by corn as affected by soil characteristics and root morphology soil. Scienty of America Journal, Madison, v. 43, n. 4, p. 880-883, 1979.

SCHWARZ, K. U.; GEISLER, G. A rapid screening method to describe genetic variability in root development of cereals. In: McMICHAEL, B. L.; PEARSON, H. (Eds.). Plant roots and their environment. Kiel: Elsevier Science, 1991. p. 632-647.

SILVA, A. E. da; GABELMAN, W. H. Screening maize inbred lines for tolerance to low $\mathrm{P}$ stress conditions. Plant and Soil, The Hague, v. 146, p. 181-187, 1992. 